Communication

\title{
Formulation of Lipoprotein Microencapsulated Beadlets by Ionic Complexes in Algae-Based Carbohydrates
}

\author{
Alexandru Vasile Rusu ${ }^{1, *}$, Florin Leontin Criste ${ }^{2}\left(\mathbb{0}\right.$, Daniel Mierliţă ${ }^{3}$, \\ Claudia Terezia Socol $1, *(\mathbb{D})$ and Monica Trif ${ }^{2, *(D)}$ \\ 1 Research and Development, CENCIRA Agrofood Research and Innovation Centre, 400650 Cluj-Napoca, Romania \\ 2 Department of Food Science, Life Science Institute, University of Agricultural Sciences and Veterinary \\ Medicine Cluj-Napoca, Science, 400372 Cluj-Napoca, Romania; florinleontincriste@gmail.com \\ 3 Nutrition and Public Health, University of Oradea, 410087 Oradea, Romania; dadi.mierlita@yahoo.com \\ * Correspondence: rusu_alexandru@hotmail.com (A.V.R.); clausocol@yahoo.com (C.T.S.); \\ monica_trif@hotmail.com (M.T.)
}

Received: 28 February 2020; Accepted: 23 March 2020; Published: 24 March 2020

\begin{abstract}
The present study aims to produce sustained-release algae-based carbohydrate microbeadlets of lipoproteins rich-in carotenoids extracted from organic sea buckthorn fruits. $\beta$-carotene represented the major compound of the lipoproteins extracts. Emulsification and algae-based carbohydrates, such as sodium-alginate and kappa-carrageenan, provide an inert environment, allowing the embedded targeted bioactive compounds-lipoproteins rich in carotenoids in our case-to maintain greater biological activity and to have a better shelf life. Furthermore, the microbeadlets prepared from sodium-alginate-kappa-carrageenan (0.75\%:0.75\% $\mathrm{w} / \mathrm{v}$ ) crosslinked with calcium ions showing $90 \%$ encapsulation efficiency have been utilized in HPMC capsules using beadlets-in-a-capsule technology, to use as a delivery system for the finished product. The GI simulated tests performed under laboratory conditions suggested that the sodium-alginate-kappa-carrageenan combination could be useful for the formulation-controlled release of microbeadlets containing lipoproteins rich in carotenoids.
\end{abstract}

Keywords: lipoprotein; carbohydrate; beadlet; emulsion; release; HPMC; beadlet-in-a-capsule

\section{Introduction}

To enhance the bioavailability of orally delivered bioactive compounds, different strategies have been investigated to assure a proper solubility and a good intestinal permeability [1-5].

Algae-based carbohydrate-encapsulating structures are continuously developed to ensure that the selected bioactives reach the lower gastrointestinal (GI) tract and establish protective mechanisms to combat metabolic syndrome. Alginate and carrageenan are additives used as texturing. These are a gelling agent and a thickener, which gives the required texture. Their ability to form a gel in the presence of calcium ions is used to produce an accurate form of a fibrous texture [6,7]. Epidemiological research data have shown that dietary supplementation with the natural carotenoids has several benefits, a correlation being made between a high carotenoid intake in the diet and a reduced risk of breast, cervical, ovarian, colorectal cancers, and cardiovascular and eye diseases. It is believed that carotenoids act in a dose- and time-dependent manner [8,9].

Solubilized lipid-based formulations are obtained to improve the gastrointestinal (GI) absorption of poorly water-soluble bioactive compounds. The nutraceutical and pharmaceutical food products are gaining market share, due to the continuous global population to age, preventive and curative [10-12]. 
The algal-based carbohydrates, alginate and kappa-carrageenan, besides the benefits of being stabilizers, thickeners or emulsifying agents, are considered to be a source of dietary fiber-an indigestible polysaccharide [13].

Ionotropic gelation is a method based on the interaction of polyelectrolytes to crosslink in the presence of divalent ions [14]. Lipoproteins are the "packages" in which cholesterol and triglycerides travel throughout the body. There are four major classes of lipoproteins, and each type helps determine a person's risk for cardiovascular disease more accurately than cholesterol measurement alone. Lipoproteins are considered to be nanoparticulate carrier systems of endogenous origin, and have the responsibility of moving the hydrophobic lipids in the blood toward different cells [15].

Studies have also shown that tumors have overexpressed low-density lipoprotein (LDL) receptors, especially in cancers, and for the proliferation lipid and cholesterol systems are required. Therefore, the key aim may be targeting therapeutics encapsulated with LDLs [16,17]. High-density lipoproteins (HDL) and LDL are naturally occurring vehicles that are attractive vessels for the delivery of bioactives and for targeting tumor cells $[18,19]$.

There is a growing market of new products containing bioactive compounds, which are the phytochemicals found in foods. They have the capacity to modulate metabolic processes resulting in the promotion of better health; therefore, there is an increasing demand for evaluation using different approaches [20,21].

The impact of chemical structures, physicochemical properties and the nature of the food matrix on the release and bioavailability of different bioactive compounds in the gastrointestinal (GI) tract have not been studied in detail. However, they are of crucial importance for the maintenance of health functions [16,17]. Targeting the bioactives to different regions of the GI is a continuous challenge for researchers, especially with regard to personalized nutrition, healthier diets and plant-based diets $[11,22]$. This is in line with the increasing efforts currently being invested to meet the consumers' expectations and needs on one hand, while on the other in finding ways to differentiate the products. It is thought that the next step in the market evolution is personalization, a personalized nutrition concept representing a new opportunity market for food companies [12].

The aim of this study has been to test how to improve solubility and bioavailability for bioactive compounds by self-emulsions, and carbohydrate-based microbeadlets to be used as controlled release carriers. The release characteristics in a simulated gastrointestinal tract of the lipoproteins contained in the self-emulsified algae-based carbohydrate microbeadlets were measured.

\section{Materials and Methods}

\subsection{Chemicals}

Natrium alginate and kappa-carrageenan were purchased from Danisco Ltd. (Cluj-Napoca, Romania). Hydroxypropyl methylcellulose (HPMC) veggie capsules $(2.5 \mathrm{~cm})$ were provided by doTerra (induced thermally (no gelling agent added)). Calcium chloride $\left(\mathrm{CaCl}_{2}\right)$, sodium dodecyl sulfate, pentanol, $\mathrm{HCl}, \mathrm{KH}_{2} \mathrm{PO}_{4}, \mathrm{NaOH}$ were purchased from Sigma-Aldrich (Darmstadt, Germany). Sanzyme (a commercial product of Pharco Pharmaceuticals, Egypt imported by Pharco Impex 93, Romania) contains papain (from Carica papaya) and pepsin, two proteolytic enzymes associated with Sanzyme 2000 (a multienzymatic complex containing proteases, amylases, lipases, celluloses and other enzymes). Triferment (a commercial product of SC BIOFARM SA, Romania), contains pancreatin.

\subsection{Extraction of Lipoproteins}

The organic fresh fruits of sea buckthorn (Hippophae rhamnoides) were purchase from a Romanian organic producer. The organic fresh fruits were washed, and broken up using a cuter, followed by centrifugation at $6000 \mathrm{rot} / \mathrm{minute}$ at $20^{\circ} \mathrm{C}$, using a sieve with $1.5 \mathrm{~mm}$ diameter holes according to the protocol described by Trif et al. [5].

The following procedure was applied: 
- Three phases-mainly a dry part, a paste and a clear juice were obtained in the first step. The organic sea buckthorn fruits seeds and skin remained in the dry part.

- The paste was centrifuged further. The three additional fractions resulted on the second step. The three fractions were called: the first fraction, which was the pellet, or the "butter" fraction; the second fraction called the lipoprotein fraction; the third fraction, called the juicy fraction.

The "butter" fraction contained a high number of lipoproteins and was used to extract the lipoproteins for further encapsulation. The lipoproteins fraction was found in a pectin network. The juicy fraction was a clear juice with a low content of lipoproteins, and was not taken into consideration for the further investigations in this study.

The "butter" fraction was washed with distillate water, and centrifuged. The lipoproteins extracted after centrifugation were self-emulsified with sodium-alginate and kappa-carrageenan to obtain the beadlets.

\subsection{Fractions Analysis and $\beta$-Carotene Quantification}

\subsubsection{Acidity Analysis}

Of the obtained fractions, the total acidity and $\mathrm{pH}$ were measured according to the protocols described by Trif et al. [5].

\subsubsection{HPLC Analysis}

For the quantification of carotenoids, the method used was HPLC carried out following the procedure described by Bindea et al., 2018 [20]. HPLC analyses for individual carotenoids were carried out on an Agilent 1200 system with a DAD detector (Agilent Tehnologies, Santa Clara, CA, USA) using a reversed phase EC 250/4.6 Nucleodur 300-5 C-18 ec. Column $(250 \times 4.6 \mathrm{~mm}), 5 \mu \mathrm{m}$ (Macherey-Nagel, Dueren, Germany). The mobile phase consisted of mixtures of acetonitrile: water ( $90 / 10 \mathrm{v} / \mathrm{v}$ (volume/volume) with $0.25 \%$ triethylamine (A), and ethyl acetate with $0.25 \%$ triethylamine (B). The gradient started with $90 \% \mathrm{~A}$ at $0 \mathrm{~min}$ to $50 \% \mathrm{~A}$ at $10 \mathrm{~min}$. The percentage of A decreased from $50 \%$ at $10 \mathrm{~min}$ to $10 \% \mathrm{~A}$ at $20 \mathrm{~min}$. The flow rate was $1 \mathrm{~mL} / \mathrm{min}$ and the chromatogram was monitored at $450 \mathrm{~nm}$. The quantity of $\beta$-carotene using a compound standard and a calibration curve was determined. Calibration curves for $\beta$-carotene were prepared at seven concentrations in the range $0-300 \mu \mathrm{g} / \mathrm{mL}$ by plotting the peak area recorded by DAD against the known concentration of the standard. The linear regression factor of the calibration curves was greater than 0.98 .

\subsubsection{UV-Vis Analysis}

The absorption spectra were recorded in a UV-Vis spectrometer (PerkinElmer, Cluj-Napoca, Romania). All measurements were performed at room temperature, and the results are the average of 3 runs. The spectrum of the major constituent of lipoproteins fraction, $\beta$-carotene, was performed in the range $300-500 \mathrm{~nm}$.

\subsection{Preparation of Lipoproteins Self-Emulsion}

Different concentrations, $0.5 \%$ weight/volume $(\mathrm{w} / \mathrm{v})$ and $0.75 \% \mathrm{w} / \mathrm{v}$ of natrium-alginate and kappa-carrageenan in a ratio 1:1 into demineralized water were dissolved by stirring for homogenization for $1 \mathrm{~h}$ at $60^{\circ} \mathrm{C}$ and let to cool down $\sim 37^{\circ} \mathrm{C}$ until a uniform and bubble free dispersion was obtained.

The obtained homogenized solution of natrium-alginate and kappa-carrageenan was mixed with the extracted lipoproteins $(5 \%$ volum/weight $(\mathrm{v} / \mathrm{w}))$ using the high shear Ultra-Turrax (Jahnke and Kunkel, IKA, Staufen, Germany) homogenizer at $5000 \mathrm{rpm}$, and blended to form oil-in-water self-emulsions. 


\subsection{Evaluation of Lipoproteins Self-Emulsion}

\subsubsection{Organoleptic Properties}

The physical aspects of self-emulsions were checked visually (homogeneity, odour and colour). The greasiness was assessed by applying it into the skin surface.

\subsubsection{Emulsion Stability}

From the prepared oil-in-water self-emulsions, an number of $20 \mathrm{~mL}$ aliquots were transferred to graded $25 \mathrm{~mL}$ cylinders, sealed, and stored for one day at room temperature. After $24 \mathrm{~h}$, the volume of the aqueous phase was measured. The stability by means of $\%$ of separation was measured according to Gangurde and Amin [23]

$$
\text { separation } \%=H_{1} / H_{0} \times 100
$$

where $H_{0}$ represents the emulsion initial height, and $H_{1}$ represents the stable emulsion after $24 \mathrm{~h}$ (upper phase height).

\subsubsection{Stability Under Centrifugation}

The self-emulsions stability was evaluated against an aggregation of emulsified lipoproteins particles. The centrifugation tubes were filled with $20 \mathrm{~mL}$ of oil-in-water self-emulsions, and further centrifuged at 10,000 rpm for $30 \mathrm{~min}$. The evaluation for the phase separation was performed [23,24].

\subsection{Microbeadlets and HMPC Capsules Containing Microbeadlets Preparation}

The method used to prepare the sea buckthorn lipoprotein-containing microbeadlets by ionotropically gelation technique was as follows: The final dispersion of oil-in-water emulsion was dropped through a $0.4 \mathrm{~mm} \times 20 \mathrm{~mm}$ syringe needle into $100 \mathrm{~mL}$ of $\mathrm{CaCl}_{2}$ concentration as a hardening bath, and was kept for 30 min under stirring to improve the mechanical strength of the beadlets, and also to prevent the aggregation of the formed ones. The formation of small beadlets took place quickly, which were collected afterwards from the hardening bath by filtration using a stainless steel sterile sieve (pore size: $0.23 \mathrm{~mm}$ ), before being transferred onto a sterile dry filter paper to absorb moisture.

Afterwards, the microbeadlets were utilized in two-piece capsule shells made from HPMC (capsule size: $2.5 \mathrm{~cm}$ ) as a delivery system, as a complete health product using beadlets-in-a-capsule technology.

\subsection{Encapsulation Efficiency}

The encapsulation efficiency ( $E E \%)$ by measuring the amount of $\beta$-carotene was calculated. The major component of the lipoprotein fraction is $\beta$-carotene. The content of $\beta$-carotene from the lipoprotein fraction, before and after encapsulation, was assayed using tetrahydrofuran (THF) spectrophotometrically at $454 \mathrm{~nm}$. To extract $\beta$-carotene from microbeadlets, the microbeadlets were crushed using a mortal and THF as solvent. The absorption spectra were recorded in a UV-Vis spectrometer as described in Section 2.3.3 [2,5].

A modified formula was used:

$$
(\mathrm{EE} \%)=\mathrm{MB} 1 /(\mathrm{D} \times \mathrm{IB} 2) \times 100
$$

where:

$M B 1=$ concentration of $\beta$-carotene in microbeadleats containing lipoproteins.

$I B 2=$ initial concentration of $\beta$-carotene in lipoproteins.

$D=$ dilution (in our case $D=1$ ). 


\subsection{Analysis of Microbeadlets}

\subsubsection{Microscopically}

The obtained microbeads' sizes and aspects were characterized with a Zeiss high performance microscope (Cluj-Napoca, Romania).

\subsubsection{Fluorescence Method}

For labelling the lipoproteins, encapsulated NBD-N-3786 (6-(7-nitrobenz-2-oxa-1,3-diazol-4-yl)amoni)hexanoyl-1-hexadecanoyl-sn-glycero-3-phosphocholine (NBD C6-HPC) fluorophores was used. A $1 \mu \mathrm{M}$ solution NBD-N-3786 solution was added to $1 \mathrm{~mL}$ lipoproteins $(5 \%)$ before being incubated at $37^{\circ} \mathrm{C}$ for $1 \mathrm{~h}$. The sample was centrifuged at $4000 \mathrm{rpm}$, the pellet collected and Tris buffer $\mathrm{pH} 8$ was added in a ratio 1:1. The sample was vortexed and microencapsulated in potassium alginate. The microbeads were controlled under a microscope with fluorescence (Zeiss, Oberkochen, Germany). The absorbance and emission were measured with the lamp with a wavelength ranging from 400 to $700 \mathrm{~nm}$.

\subsubsection{Scanning Electron Microscopy}

The physical surface and morphology of the microbeadlets were measured using a scanning electron microscope (Hitachi S-2700, iMOXS micro-X-ray fluorescence spectrometer with BSE detector, Cluj-Napoca, Romania). Microbeadlets were sputtered with gold prior to scanning, and afterwards scanned at an accelerating voltage of $15 \mathrm{kV}$.

\subsection{In Vitro simulation of Gastrointestinal Model}

For the in vitro simulation of gastrointestinal tests, a combination of the methods described by Trif et al. and Yang, and Chiang, P.-Y was adapted accordingly [5,25]. The stability of the HPMC capsules containing lipoprotein microbeadlets was measured by an evaluation of the physical integrity in different media miming biological fluids with different $\mathrm{pH}$ for $24 \mathrm{~h}$ at $37^{\circ} \mathrm{C}$ under laboratory conditions.

The simulation of gastrointestinal (GI) transit conditions under laboratory conditions was performed as per the following scheme (Figure 1):

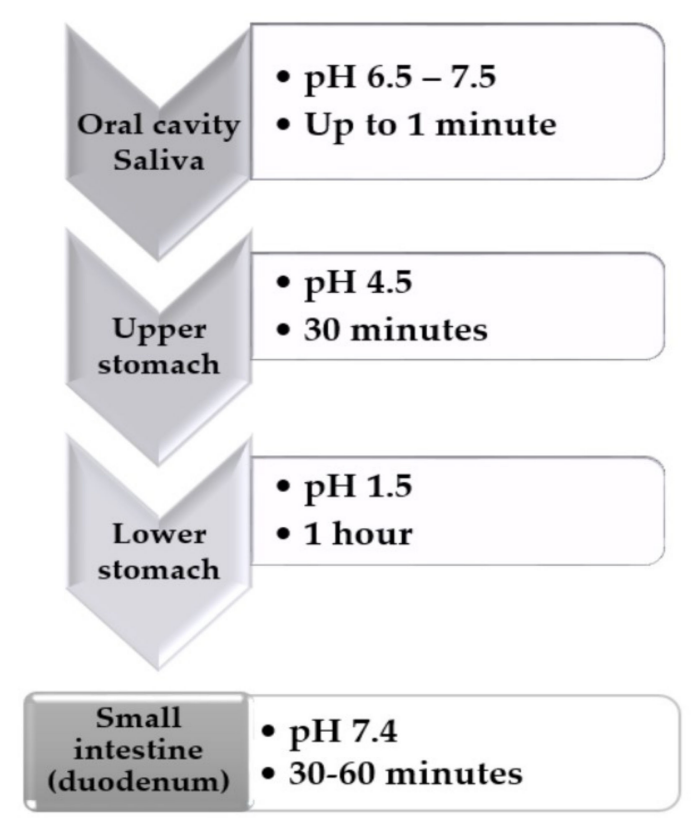

Figure 1. Schematic representation tests in different media miming biological fluids.

The conditions of simulated of GI transit was achieved by using different dissolution media: 
- $\quad \mathrm{pH} 1.5$ - consisted of $0.1 \mathrm{~N} \mathrm{HCl}$ and $5 \mathrm{~mL}$ Sanzyme (enzyme syrup containing $80 \mathrm{mg}$ papain, $40 \mathrm{mg}$ pepsin and $10 \mathrm{mg}$ Sanzyme 2000); $\mathrm{pH}$ adjusted to $1.5 \pm 0.1$.

- $\quad$ pH 4.5 - prepared by mixing SGF pH 1.5 and SIF

- $\mathrm{pH} 7.4$ in a ratio 39:61; $\mathrm{pH}$ adjusted to $4.5 \pm 0.1$. pH 7.4-consisted of $\mathrm{KH}_{2} \mathrm{PO}_{4} 1.074 \mathrm{~g}$ in $30 \mathrm{~mL}$ of $0.2 \mathrm{~N} \mathrm{NaOH}$, and one tablet of Triferment (containing pancreatin $275 \mathrm{mg}$, equivalent to a minimum enzyme activity of 2970 amylase units, 3720 lipase units and 250 protease units); $\mathrm{pH}$ adjusted to $7.4 \pm 0.1$.

The experiment was performed into an incubator with a continuous supply of carbon dioxide at $37^{\circ} \mathrm{C}$. The beadlets-in-HMPC capsule and afterwards only the beadlets, were transferred between different media miming biological fluids once after the contact time was over in each media as represented in Figure 1.

Sanzyme syrup is a commercially available product, a recommended balanced combination of digestive enzymes with specific actions, contributing to the complete digestion of food components (proteins, lipids, carbohydrates, and plant fibers).

\subsection{Statistical Analysis}

Results were expressed as a mean value with its standard deviation (mean \pm S.D.) of each sample that is repeated three times $(n=3)$. Statistical analysis was performed with Student's t-test and differences were considered as significant at $\mathrm{p}$-values under 0.05 .

\section{Results}

\subsection{Characterization of Fractions}

\subsubsection{Acidity Analysis}

The acidity was expressed as malic acid, and was in the range $1.82 \%-1.9 \%$. On the other side, the obtained fractions had a $\mathrm{pH}$ value ranging $2.7-3.1 \pm 0.2$. The $\mathrm{pH}$ of fruit juices is generally low, ranging from $\mathrm{pH} 2.5$ to $\mathrm{pH} 3.7$, and it is necessary to be checked as the acid tolerance in the juices is of high importance for further applications.

\subsubsection{Composition of Lipoproteins Fraction Used for Encapsulation by HPLC}

The $\beta$-carotene from the HPLC chromatogram was measured as a percentage of the resulting area. The calibration curve was prepared using $\beta$-carotene as the major compound of the lipoprotein fraction as standard, showing that the total content of $\beta$-carotene from the lipoprotein fraction was $10 \%$ from the total carotenoids content (estimative $4.5 \mathrm{mg} \backslash 100 \mathrm{~g}$ ). The presence of the different carotenoids (such as apocarotenoids, lutein, $\beta$-cryptoxanthin, zeaxanthin) besides $\beta$-carotene was notable.

\subsection{Self Emulsions Characterization}

The self-emulsions were orange in color and found to be smooth, and free from grittiness when applied on the skin surface. The formulations were evaluated for their stability under centrifugation. It was observed that the formulation with sodium-alginate-kappa-carrageenan $(0.5 \%: 0.5 \% \mathrm{w} / \mathrm{v})$ showed an upper phase separation of $10 \%$, and the formulation with sodium-alginate-kappa-carrageenan $(0.75 \%: 0.75 \% \mathrm{w} / \mathrm{v})$ showed no creaming or phase separations under centrifugation; therefore, it was decided to continue to use this formulation in our study.

The light microscopy imaging of the oil-in-water self-emulsion of lipoproteins and sodium-alginate-kappa-carrageenan $(0.75 \%: 0.75 \% \mathrm{w} / \mathrm{v})$, reveals the presence of lipoproteins droplets, whose size varies between $10-60 \mu \mathrm{M} \pm 0.15$ (Figure 2). 


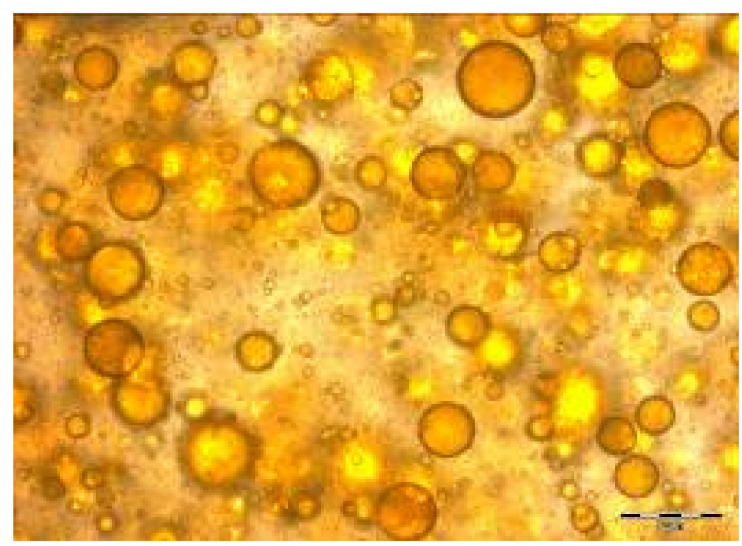

Figure 2. Self-emulsion of lipoproteins and sodium-alginate-kappa-carrageenan (0.75\%:0.75\% w/v).

The scale bar represents $100 \mu \mathrm{m}$.

\subsection{Characterization of Microbeadlets Containing Lipoproteins Encapsulated}

\subsubsection{Encapsulation Efficiency (EE\%)}

The encapsulation efficiency was $90 \%$ determined based on UV-Vis spectroscopy analysis of the content of $\beta$-carotene as the major compound from the lipoprotein fraction before and after encapsulation. The initial $\beta$-carotene content in the lipoproteins extracted fraction was $2.035 \pm 0.1 \mathrm{mg} / \mathrm{DW}$. After encapsulation, the content in the sodium-alginate-kappa-carrageenan (0.75\%:0.75\% w/v) microbeadlets was $1.830 \pm 0.1 \mathrm{mg} / \mathrm{DW}$.

\subsubsection{Microbeadlets Characterization}

The fluorescencent method was applied to show the microbeadlets' content. Firstly, the lipoproteins were labelled with the fluorescent NBD-N-3786, encapsulated and then subjected to microscopy (Figure 3a). The orange fluorescent inner tail of the microbeadlets can be observed, which are the lipoproteins labeled with NDB-N-37862. The microbeadlets' wall is green, due to the fact that NDB-N-37862 does not label polysaccharides such as alginate and carrageenan. The microbeadlets with a red core can be explained due to the thick diameter of the microbeadlets, through which the light must pass.

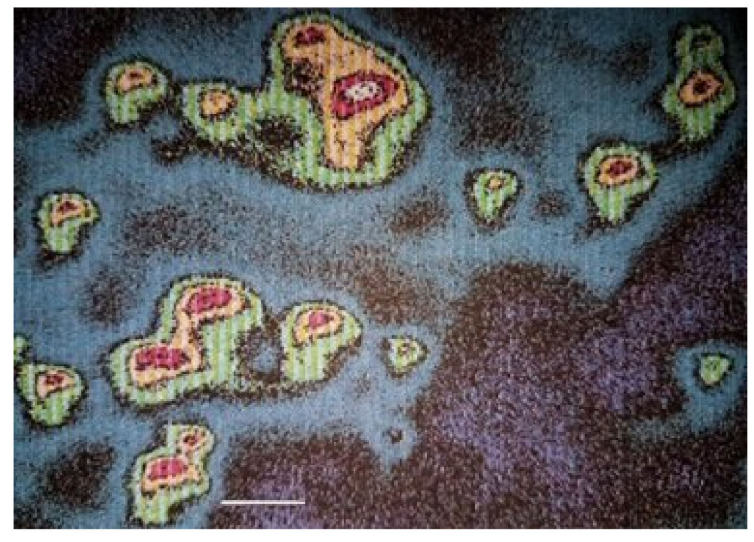

(a)

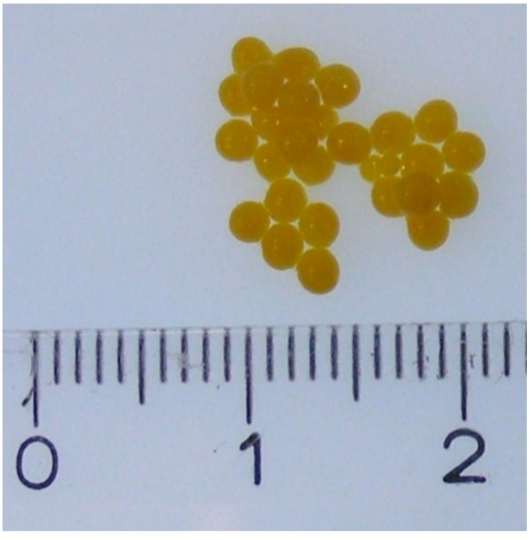

(b)

Figure 3. (a) Microbeadlets with fluorescence method. The scale bar represents $3 \mathrm{~mm}$; (b) Microbeadlets containing lipoproteins.

The microbeadlets have a size distribution ranging from 2 to $3 \mathrm{~mm} \pm 0.2$, depending on the needle used. The shape is closed to spherical and the surface is smooth (Figure $3 b$ ). 
The surface of microbeadlets obtained were irregular-this is due to the lipoprotein dispersion all over the internal structure (Figure 4a). The SEM pictures of microbeadlets show a non-porous surface, and a smooth surface morphology is exhibited but non regular due to the presence of lipoproteins dispersed all over the internal structure (Figure 4b). Several studies have shown that kappa-carrageenan enhances the thermostability of the one-polysaccharide network [26].

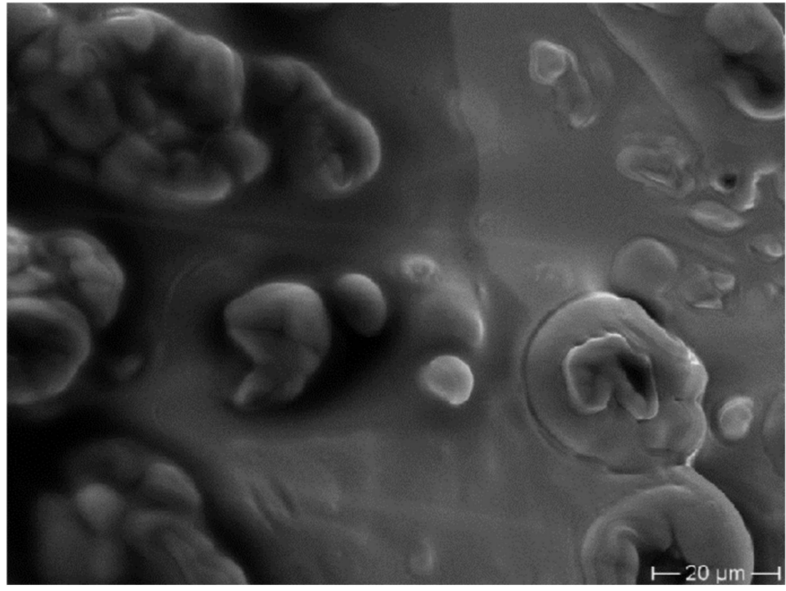

(a)

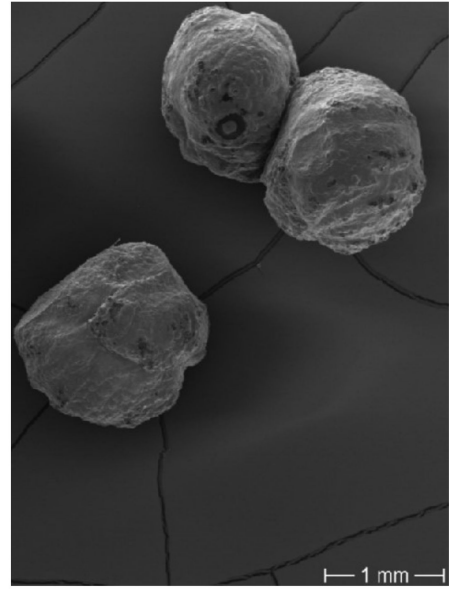

(b)

Figure 4. Scanning electron micrographs of: (a) the surface morphology of microbeadlets. The scale represents $20 \mu \mathrm{m}$; (b) microbeadlets external structure. The scale represents $1 \mathrm{~mm}$. Magnification 70×.

As cross-linking agent $\mathrm{CaCl}_{2}$ containing acetic acid glacial was used in optimum concentration $1 \mathrm{mM}$, resulting in microbeadlets with hard walls.

Sodium-alginate and kappa-carrageenan lead to the cross-linking and aggregation of both when in contact with calcium chloride, and due to exchange of divalent ions of calcium $\left(\mathrm{Ca}^{++}\right)$during the reticulation process, the creation of a strong network, which in turn leads to a very strong matrix (Figure 5).

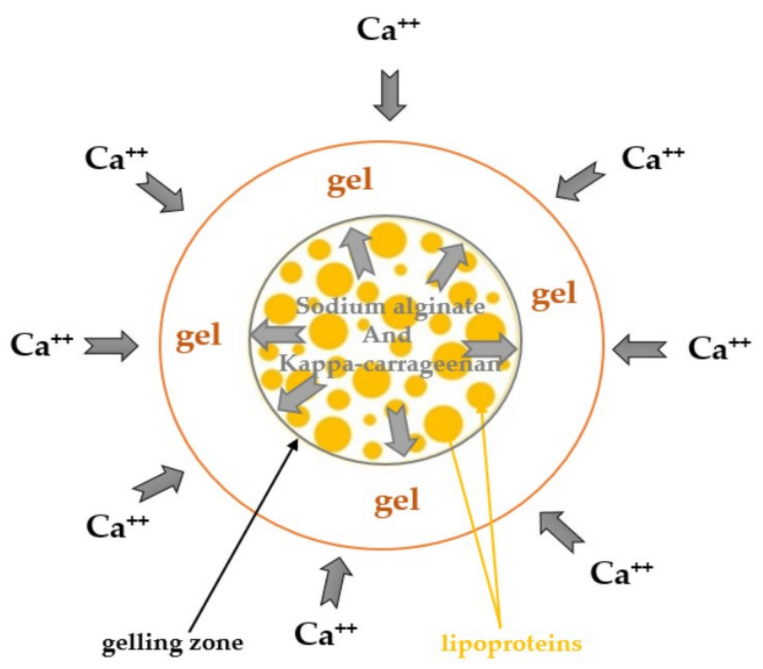

Figure 5. Cross-linking schematic process of sodium-alginate-kappa-carrageenan in the presence of calcium ions via diffusion method.

The cross-linking ions are possible because of the two basic methods for a sodium-alginate-kappa-carrageenan gel preparation: firstly, the diffusion method, and secondly the internal setting method. In the diffusion method, the cross-linking ions are able to diffuse from a large 
outer reservoir into a sodium-alginate-kappa-carrageenan alginate solution (Figure 5). This diffusion permits a rapid kinetic gelling. In general, its utilization is well known for immobilization purposes, resulting in one single gel bead with entrapped bioactives. A high-speed setting has a great advantage, as when restructuring foods, the given size and shape of the final product is a target.

\subsection{HMPC Capsules Containing Microbeadlets with Lipoproteins}

For further nutraceutical, dietary and nutritional supplements applications, the food-grade and vegetable-derived microbeadlets have been utilized in HPMC capsules as a delivery system, as complete health products for a specific need approved for internal use using beadlets-in-a-capsule technology (Figure 6). An extra advantage of the thermally gelled two-piece HPMC capsules used in the present study is the unaffected release performance, due to the presence of calcium ions [27] and the cross-linking of sodium-alginate-kappa-carrageenan microbeadlets.

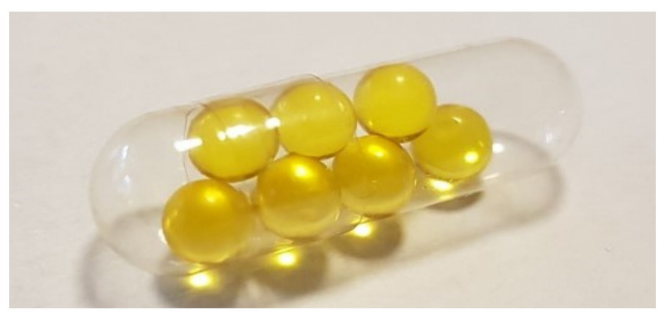

(a)

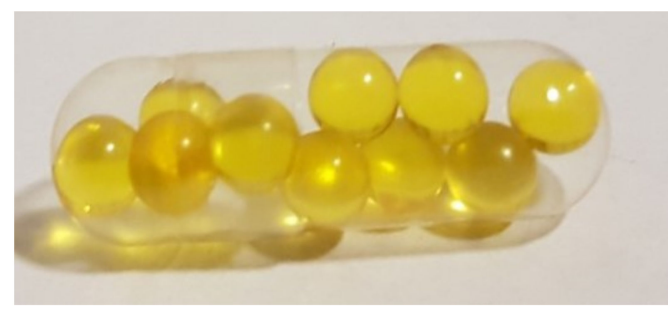

(b)

Figure 6. (a) and (b) Microbeadlets with lipoproteins incorporated in two-piece capsule shells made from HPMC (capsule size: $2.5 \mathrm{~cm}$ ) using beadlets-in-a-capsule technology.

Carbohydrates from algae such as sodium-alginate and kappa-carrageenan-and HPMC capsules (cellulose fiber)—are considered food additives in accordance with EC regulation No. 1333/2008, and are approved for vegetarians by the Vegetarian Society [28]. Therefore, they are increasingly favored as free animal by-products, due to their properties being preferred for moisture-sensitive active bioactive compounds, as well as for the "natural" image the nutritional supplement industry can confer on the product, expanding the new possibilities for marketing purposes and making it eligible for organic label language (EU), and suitable for use with other organic bioactive ingredients extracted as lipoproteins from organic sea buckthorn fruits.

\subsection{Stability in Different Simulated Gastrointestinal Fluids under Laboratory Conditions}

In our study, the HMPC capsules containing microbeadlets with lipoproteins, obtained using beadlets-in-a-capsule technology (Figure 6), were dissolved in simulated gastric fluid (SGF) of pH 4.5 and microbeadlets were released Figure 7B.). Furthermore, the microbeadlets were expose to the simulation of gastrointestinal (GI) transit conditions under the described laboratory conditions (Figure 7B-D): 


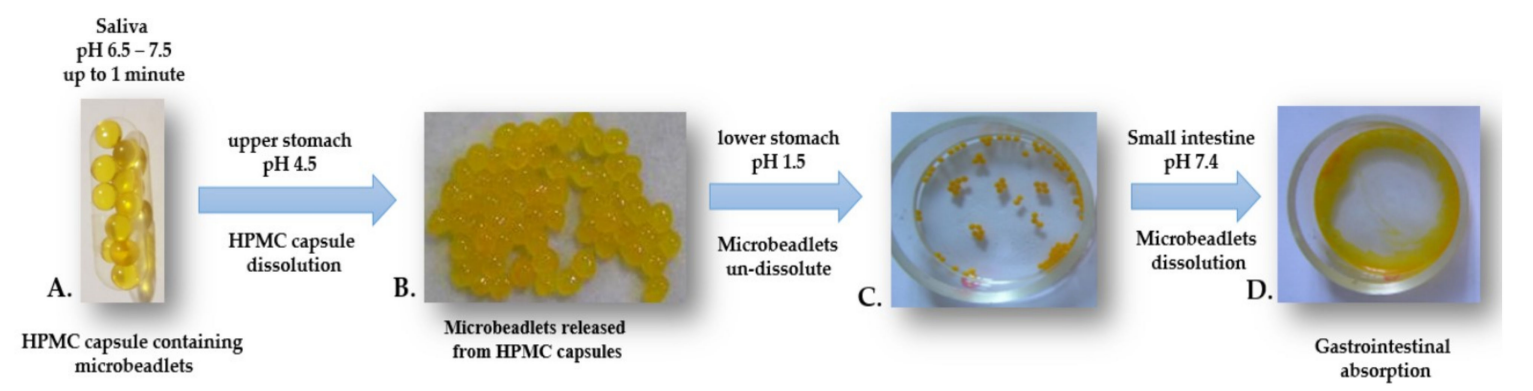

Figure 7. Stability in different simulated gastrointestinal fluids under laboratory conditions: (A) microbeadlets containing lipoproteins-in-a HPMC capsule; (B) Microbeadlets released from HMPC capsule after its dissolution at $\mathrm{pH} 4.5$; (C) Microbeadlets remain un-dissolved at $\mathrm{pH}$ 1.5; (D) Microbeadlets completely dissolved at $\mathrm{pH} 7.4$.

Along the GI tract, the $\mathrm{pH}$ varies. In the first section of the GI tract, the oral cavity, the $\mathrm{pH}$ of saliva is usually between 6.5-7.5, and last up to $1 \mathrm{~min}$ (Figure 7A.). In any dissolution medium with a $\mathrm{pH}$ below or equal to 5.8, HPMC capsule shells dissolved rapidly. Therefore, in the oral cavity, the HPMC capsules remain intact, and could be taken with cold or warm drinks.

The HPMC capsules then access the upper part of the stomach, which has a pH between 4.0-6.5, before the pre-digestion, where they begin to be dissolved, releasing the microbeadlets (Figure 7B). Furthermore, in the lower part of the stomach where the secretion of hydrochloric acid (HCI) and pepsin occur, the $\mathrm{pH}$ is between 1.5-4.0. The microbeadlets remain in the lower part of the stomach undissolved (Figure 7C). After entering the duodenum (small intestine), the $\mathrm{pH}$ changes to 7.0-8.5. The microbeadlets exhibit visual signs of swelling and erosion. As expected, the swelling volumes of the microbeadlets increased by increasing the $\mathrm{pH}$. At $\mathrm{pH}>7$ the microbeadlets were completely dissolved as shown in the Figure 7D. According to several studies and supported by different health societies, $90 \%$ of the nutrients absorption taken in by the body occurs in the small intestine (Canadian Cancer Society).

\section{Discussion}

The carotenoids, as a supplement, have to be presented in a ready-absorbed form through the gastrointestinal tract to be able to exert their function on a targeted part of the human body. Commercially available carotenoid supplements are in the form of soft gel capsules obtained from gelatin as soft shell or two-piece hard gel capsules. Nowadays, the attention is focused on developing products suitable for vegetarians and vegans that are also allergen-free [29].

Carbohydrates from algae and plant-based bioactive ingredients such as carotenoids are of interest. In our study, the lipoproteins rich in carotenoids as bioactive ingredients were encased in microbeadlets obtained from carbohydrate algae-based ionic complexes.

The lipoprotein self-emulsions were obtained without adding an emulsifier. We examined this possibility, bearing in mind the presence of pectin as structural natural matrix found in sea buckthorn fruits (can be noticed the presence of in Figure 2). The extraction of free-pectin matrix lipoproteins using green methods cannot be achieved. Therefore, the presence of some remaining pectin surrounding the lipoproteins influences the stability of an emulsion—being considered a natural emulsifier [30,31].

The size of the injector plays a decisive role in the formation of microbeadlets, which further influences the final filling of the HPMC capsules. When using a $0.4 \mathrm{~mm} \times 20 \mathrm{~mm}$ size injector, the capacity of HPMC capsules could not be filled completely by the microbeadlets (Figure 6). Further research will address the evaluation of smaller injectors, and their capacity to produce stable microbeadlets capable to completely fill the HPMC capsule.

The carotenoids absorption is facilitated by fats [32]; therefore, the system of lipoproteins fraction extracted from sea buckthorn encased in microbeadlets makes this system an ideal supplement formulation, and a much higher bioavailability of carotenoids is expected. Besides, following the 
enhancement of carotenoids' stability, their physical properties are considerably improved due to the lipoproteins and the encapsulation technique used. The obtained microbeadlets are suitable for use in food supplements and incorporated in food products, and for further medical applications. A $\beta$-carotene of up to $50 \%$ superior bioavailability has been given by encapsulation of it in beadlets than in oil suspension in softgel capsules [33,34].

The encapsulation of a well-known anticancer agent curcumin with lipoproteins as natural vehicles has been attractive for bioactives delivery and targeting tumor cells, and has shown an improved anticancer treatment $[35,36]$.

Sodium-alginate and kappa-carrageenan have been investigated as dietary supplements with health benefits, and have been incorporated in different products showing amazing results, such as reducing the blood sugar level (glucose absorption rates reduced) [37,38]. Even compared with other dietary carbohydrates such as guar gum in products like snack bars, sodium-alginate has reduced postprandial peak glucose concentrations and total glucose uptake over $3 \mathrm{~h}$ [39-41].

The proposed cross-linked combination of algae-based carbohydrates, sodium-alginate and kappa-carrageenan, can be considered as a new method for the loading of plant bioactives and controlled delivery systems. Their further utilization in HPMC capsules as a delivery system in a complete product make them a good candidate for nutraceutical, and dietary and nutritional supplement applications. Nowadays, the naturally occurring biomaterials (biopolymers) and the bioactives extracted from organic plants are economically attractive and an innovative approach for the food industry [42] and other practical applications (following the advantages of the capsule-in-a-capsule technology, tablet-in-a-capsule technology or duo-capsule) [43], especially in personalized food and feed diets, and medical nutrition [11,12,16,44-46].

\section{Conclusions}

In this study, a successful lipoprotein fraction-rich in carotenoids and extracted from organic sea buckthorn fresh fruits-has been encapsulated into a mixture of algae-based carbohydrates (sodium-alginate-kappa-carrageenan, ratio 1:1), in microbeadlets obtained using a crosslinking method with calcium chloride. A 90\% encapsulation efficiency has been achieved. The microbeadlets have been utilized in HPMC capsules, as a complete product delivery system, for further nutraceutical, and dietary and nutritional supplement applications.

The GI simulated tests performed under laboratory conditions suggested that the sodium-alginate-kappa-carrageenan combination could be useful for the formulation and controlled release of microbeadlets containing lipoproteins rich-in carotenoids. This represents potential further applications, enabled by the use of the microbeads either in their present form or as duo-capsule. Through the beadlets-in-a-capsule method applied, the bioactives can be easily targeted to different regions of the GI, with potential implications in personalized nutrition and plant-based diets.

Author Contributions: Conceptualization, A.V.R. and M.T.; methodology, M.T.; validation, F.L.C., D.M. and C.T.S.; formal analysis, M.T. and A.V.R.; investigation, M.T., A.V.R., F.L.C., D.M and C.T.S.; data curation, M.T.; writing-original draft preparation, M.T. and A.V.R.; writing—review and editing, M.T., F.L.C., D.M., A.V.R. and C.T.S.; visualization, M.T.; supervision, M.T.; project administration, C.T.S.; funding acquisition, M.T., A.V.R. and C.T.S. All authors have read and agreed to the published version of the manuscript.

Funding: This research was funded by a grant of the Romanian National Authority for Scientific Research and Innovation, CCDI-UEFISCDI, project number 27/2018 CO FUND-MANUNET III-NON-ACT-2, within PNCDI III, and ERA-NET MANUNET - II TOMATOCYCLE developed with the support of UEFISCDI (2017-2020).

Conflicts of Interest: The authors declare no conflict of interest. The funders had no role in the design of the study; in the collection, analyses, or interpretation of data; in the writing of the manuscript, or in the decision to publish the results. 


\section{References}

1. Irimie, A.I.; Sonea, L.; Jurj, A.; Mehterov, N.; Zimta, A.A.; Budisan, L.; Braicu, C.; Berindan-Neagoe, I. Future trends and emerging issues for nanodelivery systems in oral and oropharyngeal cancer. Int. J. Nanomed. 2017, 26, 4593-4606. [CrossRef] [PubMed]

2. Trif, M.; Socaciu, C. Evaluation of effiency, release and oxidation stability of seabuckthorn microencapsulated oil using Fourier transformed infrared spectroscopy. Chem. Listy 2008, 102, s1198-s1199.

3. Zanoaga, O.; Braicu, C.; Jurj, A.; Rusu, A.; Buiga, R.; Berindan-Neagoe, I. Progress in Research on the Role of Flavonoids in Lung Cancer. Int. J. Mol. Sci. 2019, 20, 4291. [CrossRef] [PubMed]

4. Obasi, T.C.; Moldovan, R.; Toiu, A.; Braicu, C.; Bodoki, E.; Berindan-Neagoe, I.; Oniga, I.; Sandulescu, R.; Oprean, R. Molecular-trapping in Emulsion's Monolayer: A New Strategy for Production and Purification of Bioactive Saponins. Sci. Rep. 2017, 6, 14511. [CrossRef] [PubMed]

5. Trif, M.; Vodnar, D.C.; Mitrea, L.; Rusu, A.V.; Socol, C.T. Design and Development of Oleoresins Rich in Carotenoids Coated Microbeads. Coatings 2019, 9, 235. [CrossRef]

6. Martins, E.; Poncelet, P.; Rodrigues, R.C.; Renard, D. Oil encapsulation techniques using alginate as encapsulating agent: Applications and drawbacks. J. Microencapsul. 2016, 34, 754-771. [CrossRef]

7. Trif, M.; Csutak, E.; Perez-Moral, N.; Gagyi, T.; Pintori, D.; Bethke, M.; Wilde, P. TeRiFiQ EU Project: Multiple Gel in Oil in Water Emulsions as Fat Replacers in Sauces and Ready Prepared Foods. Bull. UASVM Food Sci. Technol. 2016, 73, 47-48. [CrossRef]

8. Braicu, C.; Gulei, D.; Raduly, L.; Harangus, A.; Rusu, A.; Berindan-Neagoe, I. Altered expression of miR-181 affects cell fate and targets drug resistance-related mechanisms. Mol. Aspects Med. 2019, 70, 90-105. [CrossRef]

9. Milani, A.; Basirnejad, M.; Shahbazi, S.; Bolhassani, A. Carotenoids: Biochemistry, pharmacology and treatment. Br. J. Pharmacol. 2017, 174, 1290-1324. [CrossRef]

10. Strasdat, B.; Bunjes, H. Incorporation of lipid nanoparticles into calcium alginate beads and characterization of the encapsulated particles by differential scanning calorimetry. Food Hydrocoll. 2013, 30, 567-575. [CrossRef]

11. Rusu, A.; Randriambelonoro, M.; Perrin, C.; Valk, C.; Alvarez, B.; Schwarze, A.-K. Aspects Influencing Food Intake and Approaches towards Personalising Nutrition in the Elderly. J. Popul. Ageing 2020. [CrossRef]

12. Trif, M.; Muresan, L.; Bethke, M. Personalised Nutritional Powder for Elderly Developed in OPTIFEL European Project. Bull. UASVM Food Sci. Technol. 2016, 73. [CrossRef]

13. Qin, Y. Health Benefits of Bioactive Seaweed Substances. Bioact. Seaweeds Food Appl. 2018. [CrossRef]

14. Henao, E.; Delgado, E.; Contreras, H.; Quintana, G. Polyelectrolyte Complexation versus Ionotropic Gelation for Chitosan-Based Hydrogels with Carboxymethylcellulose, Carboxymethyl Starch, and Alginic Acid. Inter. J. Chem. Eng. 2018. [CrossRef]

15. Shukla, T.; Upmanyu, N.; Pandey, S.P.; Sudheesh, M. Site-specific drug delivery, targeting, and gene therapy. In Nanoarchitectonics in Biomedicine; Elsevier BV: San Diego, CA, USA, 2019; pp. 473-505. [CrossRef]

16. Braicu, C.; Mehterov, N.; Vladimirov, B.; Sarafian, V.; Nabavi, S.M.; Atanasov, A.G.; Berindan-Neagoe, I. Nutrigenomics in cancer: Revisiting the effects of natural compounds. Semin Cancer Biol. 2017, 46, 84-106. [CrossRef]

17. Harisa, G.I.; Alanazi, F.K. Low density lipoprotein bionanoparticles: From cholesterol transport to delivery of anti-cancer drugs. Saudi Pharm. J. 2014, 22, 504-515. [CrossRef]

18. Chaudhary, J.; Bower, J.; Corbin, I.R. Lipoprotein Drug Delivery Vehicles for Cancer: Rationale and Reason. Int J. Mol. Sci. 2019, 20, 6327. [CrossRef]

19. Cojocneanu Petric, R.; Braicu, C.; Raduly, L.; Zanoaga, O.; Dragos, N.; Monroig, P.; Dumitrascu, D.; Berindan-Neagoe, I. Phytochemicals modulate carcinogenic signaling pathways in breast and hormone-related cancers. Onco Targets Ther. 2015, 8, 2053-2066. [CrossRef]

20. Bindea, M.; Rusu, B.; Rusu, A.; Trif, M.; Leopold, L.F.; Dulf, F.; Vodnar, D.C. Valorification of crude glycerol for pure fractions of docosahexaenoic acid and $\beta$-carotene production by using Schizochytrium limacinum and Blakeslea trispora. Microb. Cell Factories 2018, 17, 97. [CrossRef]

21. Dietrich, T.; Del Carmen Villaran Velasco, M.; Echeverría, P.J.; Pop, B.; Rusu, A. Crop and Plant Biomass as Valuable Material for BBB. Alternatives for Valorization of Green Wastes. In Biotransformation of Agricultural Waste and By-Products: The Food, Feed, Fibre, Fuel (4F) Economy; Elsevier: San Diego, CA, USA, 2016. 
22. Petrut, R.F.; Danthine, S.; Blecker, C. Assessment of partial coalescence in whippable oil-in-water food emulsions. Adv. Colloid Interface Sci. 2016, 229, 25-33. [CrossRef]

23. Gangurde, A.B.; Amin, P.D. Microencapsulation by Spray Drying of Vitamin A Palmitate from Oil to Powder and Its Application in Topical Delivery System. J. Encapsulation Adsorpt. Sci. 2017, 7, 10-39. [CrossRef]

24. Patil, A.K.; Gangurde, A.B.; Amin, P.D. Comparative Study of Different Formulations of Aceclofenac as a Topical Drug Delivery System and It's in Vitro and in Vivo Characterization. Int. J. Pharm. Sci. Res. 2014, 5, 3401.

25. Yang, K.-M.; Chiang, P.-Y. Preparation and Evaluation of Release Formulation of $\gamma$-Oryzanol/Algae Oil Self-Emulsified with Alginate Beads. Mar. Drugs 2019, 17, 156. [CrossRef] [PubMed]

26. Mohamadnia, Z.; Zohuriaan-Mehr, M.J.; Kabiri, K.; Jamshidi, A.; Mobedi, H. Ionically cross-linked carrageenan-alginate hydrogel beads. J. Biomater. Sci. Polym. Ed. 2008, 19, 47-59. [CrossRef] [PubMed]

27. Gullapalli, R.P. Dissolution of Liquid-Filled Capsules Based Formulations. Ch. 18 in Poorly Soluble Drugs: Dissolution and Drug Release. In PanStanford Series on Pharmaceutical Analysis; Webster, G.K., Jackson, J.D., Bell, R.G., Eds.; Pan Stanford Publishing Pte. Ltd.: Singapore, 2017.

28. Sutapa, M. HPMC as capsule shell material: Physicochemical, pharmaceutical and biopharmaceutical and biopharmaceutical properties. Int. J. Pharm. Pharm. Sci. 2017, 9. [CrossRef]

29. Chiwele, I.; Jones, B.E.; Podczeck, F. The shell dissolution of various empty hard capsules. Chem. Pharm. Bull. (Tokyo) 2000, 48, 951-956. [CrossRef]

30. Ngouémazong, E.D.; Christiaens, S.; Shpigelman, A.; Van Loey, A.; Hendrickx, M. The Emulsifying and Emulsion-Stabilizing Properties of Pectin: A Review. Compr. Rev. Food Sci. Food Saf. 2015, 14. [CrossRef]

31. Verkempinck, S.H.E.; Kyomugasho, C.; Salvia-Trujillo, L.; Denis, S.; Bourgeois, M.; Van Loey, A.M.; Hendrickx, M.E.; Grauwet, T. Emulsion stabilizing properties of citrus pectin and its interactions with conventional emulsifiers in oil-in-water emulsions. Food Hydrocoll. 2018, 85, 144-157. [CrossRef]

32. Britton, G.; Liaaen-Jensen, S.; Pfander, H. (Eds.) Carotenoids Volume 5: Nutrition and Health; Birkhäuser: Basel, Switzerland, 2009. [CrossRef]

33. Fuller, C.J.; Butterfoss, M.S.; Failla, M.L. Relative bioavailability of betacarotene from supplement sources. Nutr. Res. 2001, 21, 1209-1215. [CrossRef]

34. Jayant, D.; Laxman, K.; Prakash, B. Stable Oil Suspensions Comprising Lipophilic Nutrient with Enhanced Bioavailability and Process of Preparation. WO2016083874A1, 2 June 2016.

35. Sanidad, K.Z.; Zhu, J.; Wang, W.; Du, Y.; Zhang, G. Effects of Stable Degradation Products of Curcumin on Cancer Cell Proliferation and Inflammation. J. Agric. Food Chem. 2016, 64, 9189-9195. [CrossRef]

36. Braicu, C.; Buse, M.; Busuioc, C.; Drula, R.; Gulei, D.; Raduly, L.; Rusu, A.; Irimie, A.; Atanasov, A.G.; Slaby, O.; et al. A Comprehensive Review on MAPK: A Promising Therapeutic Target in Cancer. Cancers 2019, 22, 1618. [CrossRef] [PubMed]

37. Jutkova, A.; Chorvat, D.; Miskovsky, P.; Jancura, D.; Datta, S. Encapsulation of anticancer drug curcumin and co-loading with photosensitizer hypericin into lipoproteins investigated by fluorescence resonance energy transfer. Int. J. Pharm. 2019, 564, 369-378. [CrossRef] [PubMed]

38. Mackie, A.R.; Macierzanka, A.; Aarak, K.; Rigby, N.M.; Parker, R.; Channell, G.A.; Harding, S.E.; Bajka, B.H. Sodium alginate decreases the permeability of intestinal mucus. Food Hydrocoll. 2016, 52, 749-755. [CrossRef] [PubMed]

39. Dettmar, P.W.; Strugala, V.; Craig Richardson, J. The key role alginates play in health. Food Hydrocoll. 2011, 25, $263-266$. [CrossRef]

40. Qin, Y. (Ed.) 9-Health Benefits of Bioactive Seaweed Substances. In Bioactive Seaweeds for Food Applications; Academic Press: Cambridge, MA, USA, 2018; pp. 179-200. [CrossRef]

41. Gillet, S.; Aguedo, M.; Petrut, R.; Olive, G.; Anastas, P.; Blecker, C.; Richel, A. Structure impact of two galactomannan fractions on their viscosity properties in dilute solution, unperturbed state and gel state. Int. J. Biol. Macromol. 2017, 96, 550-559. [CrossRef]

42. Mitrea, L.; Călinoiu, L.-F.; Martău, G.-A.; Szabo, K.; Teleky, B.-E.; Mureșan, V.; Rusu, A.-V.; Socol, C.-T.; Vodnar, D.-C. Poly(vinyl alcohol)-Based Biofilms Plasticized with Polyols and Colored with Pigments Extracted from Tomato By-Products. Polymers 2020, 12, 532. [CrossRef]

43. Lengyel, M.; Kállai-Szabó, N.; Antal, V.; Laki, A.J.; Antal, I. Microparticles, Microspheres, and Microcapsules for Advanced Drug Delivery. Sci. Pharm. 2019, 87, 20. [CrossRef]

44. Mierlita, D. Fatty acids profile and oxidative stability of eggs from laying hens fed diets containing hemp seed or hempseed cake. S. Afr. J. Anim. Sci. 2019, 49. [CrossRef] 
45. Forstner, S.; Rusu, A. Development of personalised food for the nutrition of elderly consumers. In Know Your Food: Food Ethics and Innovation; Academic Publishers: Wageningen, The Netherlands, 2015; pp. $24-27$. [CrossRef]

46. Criste, F.L.; Mierlita, D.; Simeanu, D.; Boisteanu, P.C.; Pop, I.M.; Georgescu, B.; Nacu, G. Study of Fatty Acids Profile and Oxidative Stability of Egg Yolk from Hens Fed a Diet Containing White Lupine Seeds Meal. Revista Chim. 2018, 69, 2454-2460. [CrossRef] article distributed under the terms and conditions of the Creative Commons Attribution (CC BY) license (http://creativecommons.org/licenses/by/4.0/). 